\title{
Towards a Framework For Testing Distributed Multimedia Software Systems
}

\author{
Vojislav B. Mišić ${ }^{1} \quad$ Samuel T. Chanson ${ }^{2} \quad$ Shing-Chi Cheung ${ }^{2}$ \\ The Hong Kong University of Science and Technology \\ ${ }^{1}$ Dept. of Information and Systems Management $\quad{ }^{2}$ Dept. of Computer Science \\ Clear Water Bay, Kowloon, Hong Kong \\ vmisic@ust.hk, \{chanson, scc\}@es.ust.hk
}

\begin{abstract}
With the advancement in network speed and computing power, distributed multimedia applications are becoming popular. However, general principles of system testing cannot be directly applied to testing of distributed multimedia software systems (DMSS) on account of their stringent timing and synchronization requirements. This paper presents a framework for testing DMSS with special emphases on test case generation and test architecture. We propose ways to greatly cut down the number of test cases without compromising the test effectiveness. Procedures for testing the servers and the clients are also discussed.
\end{abstract}

Keywords: multimedia software, distributed systems, testing, temporal relationships

\section{Introduction}

Multimedia software is a general term for applications which make use of various information media, such as audio, video, animation, images, and text, in an integrated presentation. Systems which run such applications can be centralized or distributed, with one or more servers, and one or more clients. The majority of multimedia systems still belong to the single-server, single-client category. Some existing applications, such as computer-aided instruction, involve a single server and multiple clients, while others (e.g., multimedia conferencing) require several servers and clients, with each system being both a server and a client at the same time. A distributed multimedia system, in its most general form, consists of a number of servers and clients (not necessarily distinct), connected by a suitable network. A distributed multimedia software system (DMSS), then, is the collection of multimedia applications which run on such systems.

The popularity of distributed multimedia systems is growing rapidly, and they are the subject of much current research. However, the development of such systems is not easy, not only because of their inherent complexity, but-for the most part-because of the very nature of their requirements, which include stringent timing and synchronization constraints. The server software must deliver the data in the form of continuously running streams; the client software must be able to receive, decode, and resynchronize the data streams, and play them through the appropriate display subsystem(s). Moreover, the DMSS should allow its users to alter almost any aspect of the presentation: spatial, functional, and temporal. All of these tasks have to be performed in real time. Consequently, common development approaches are often inappropriate, which means that most, if not all, development phases-including testing-must be substantially modified. However, current work on the testing of multimedia systems has been mainly geared towards testing of the underlying network (e.g., [7, 8]), and testing of the quality of the presentation contents (e.g., [12]). Testing of multimedia software, in contrast, has received little attention, and even then it has been tackled at a conceptual level orly [14].

It should be stressed that, despite some superficial similarities, DMISS testing simply cannot be treated as a special case of software testing. For example, protocol testing [15] deals with the network and associated protocols, and makes heavy use of formal specification techniques. Yet, in protocol testing, the exact timing of events is rarely needed - it is only their order that is important, while in DMSS testing both the order of events and their precise timing are relevant.

Another type of testing that comes to mind is performance testing, which often relies on the measurement of time, and some of the tests may indeed be useful in multimedia testing. However. performance is usually tested system-wide, using measures which are essentially statistical in nature, such as system throughput and response time. On the contrary, testing of a DMSS is predominantly event-related and requires 
measurements at a very detailed level, e.g., the timing of individual frames and synchronization of frame streams.

Our research attempts to define a general framework for testing distributed multimedia software systems by identifying the specific requirements of such systems, and then relating these requirements to the design of test architecture and test case generation. Once such a framework is established, it can be used as the basis for detailed definitions of other aspects of the testing methodology.

The paper is organized in the following manner. Section 2 presents some pertinent characteristics of multimedia data and multimedia applications, in particular those relevant to testing. A simple classification of errors is outlined in Section 3 together with the associated classification of tests. The next Section presents a general architecture for DMSS testing and elaborates on the structure of the tester. It is shown that separate testing of server and client systems is not only possible, but actually advantageous from the controllability point of view. Section 5 discusses test case selection issues with the objective of reducing the number of test cases while retaining the effectiveness of the test. Procedures for testing both the server and client systems are outlined in Section 6. Finally, Section 7 concludes the work and outlines some directions for further research.

\section{On multimedia data}

Multimedia data are either generated in real-time, or come from a pre-orchestrated data source (server). During the course of a presentation, four distinct phases may be observed.

First, the connection between the server(s) and the client(s) is established, and the actions required for the communication (invoking appropriate applications, and opening of necessary protocol ports) are performed. Note that DMSS generally requires a connection-oriented protocol in order to guarantee the correct time ordering of the frames.

A presentation system (client), under user control; requests the data from the appropriate server(s). After the schedule of the presentation is negotiated, the server retrieves the desired data, produces the data streams and sends them over the network to the requesting client. The client then 'assembles' the presentation and presents it to the users.

In some applications such as multimedia conferencing, dynamic reconfiguration (because of new participants joining or leaving the session) may be required.

Finally, after the end of the presentation, the connection between the client and server is terminated.

The actual presentation consists of several components (or component objects) whose temporal relationships are prescribed in advance by the author of the presentation. The component-level schedule of a multimedia presentation is often represented with the so-called timeline diagrams, in
Table 1. Temporal relationship between two multimedia objects.

\begin{tabular}{|l||l|l|}
\hline & absolute timing & relative timing \\
\hline \hline \multirow{3}{*}{ implicit } & A equals B & A during B \\
\cline { 2 - 3 } & A meets B & A before B \\
\cline { 2 - 3 } & A starts with B & A earlier_than B \\
\cline { 2 - 3 } & A ends with B & A ahead_of B \\
\hline explicit & A before B by $t$ & \\
\cline { 2 - 3 } & A earlier_than B by $t$ & \\
\cline { 2 - 3 } & A ahead_of B by $t$ & \\
\hline
\end{tabular}

which the time increases along the $x$-axis while the individual components of a presentation are shown along the $y$-axis. Following Allen [1], the temporal relationships between individual components may be expressed with the operators listed in Table $1^{1}$, some of which include absolute time intervals, while others use relative timing, i.e., express order relations only. Other specification techniques are also possible; Fig. 1 shows three different specifications of the same presentation.

Some presentation components are stream-oriented (audio and video being the most common), consisting of a sequence of frames which are decoded at the client and processed by the appropriate 'display' subsystem. (A frame, in the context of this paper, means 'a digitized sample, or a collection of digitized samples'.) The frames within a stream must be played back at a speed defined by the appropriate quality requirements: for example, a specified number of frames per second must be displayed for a video stream. Sometimes, frames contain data from multiple streams, usually a video and the associated audio stream(s); this is the case with the widely accepted MPEG2 standards [10]. Different streams have different tolerances with regard to frame jitter and frame ioss, wïin audio streams being the most critical. while video streams are generally more tolerant. Nonstream objects, such as text and still images, are much less demanding in terms of bandwidth requirements; however, text does not tolerate any data loss at all.

Besides the timing constraints specific to each stream, different streams that comprise the presentation must be synchronized to obtain the desired effect. Some streams must be naturally synchronized (as in the case of multimedia conferencing), while others are specified by the author of the presentation [13]. For high-bandwidth streams, such as

\footnotetext{
'The operator 'before' as in 'A before B' means 'the object $A$ has ended before the object B started" [4]. Similarly, 'A earlier hhan B' means the component $A$ has started before the stan of the component $B$. and 'A ahead_of $B$ ' means that the component $A$ has ended before the end of component B.
} 


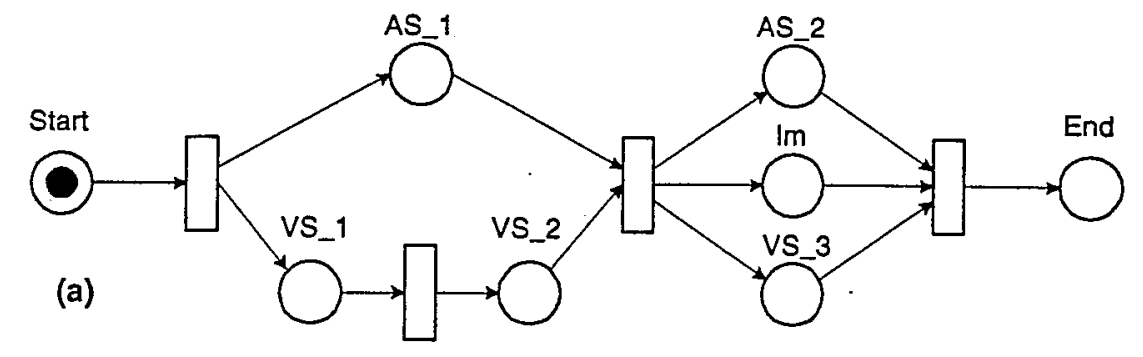

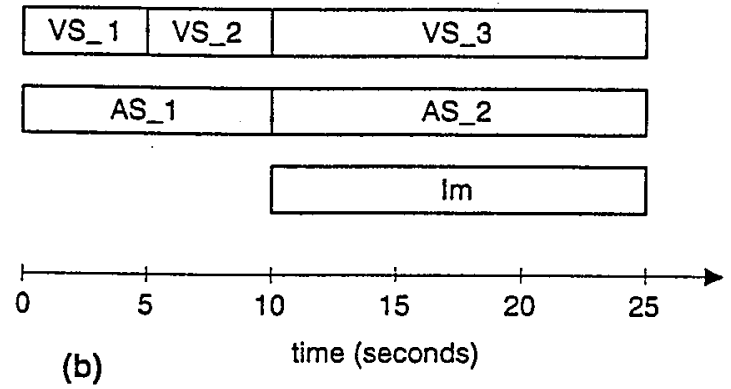

(b)

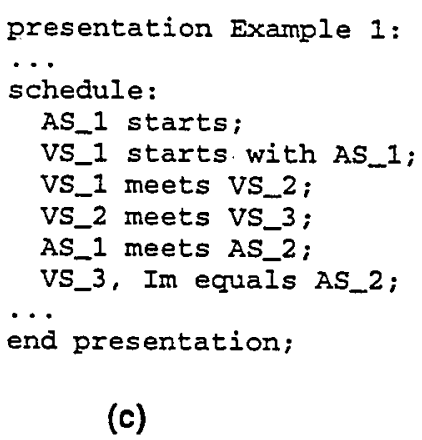

(c)

\section{Figure 1. Specifications of an example presentation: (a) Petri net, (b) timeline diagram, and (c) component temporal relationships.}

audio and video, synchronization must be effected at the frame level; for low-bandwidth data, such as still images and text, synchronization at the object level usually suffices.

In a multiple-stream presentation, one of the streams (usually an audio stream) is used as a reference (master), to which all others are synchronized. Audio and video data contained within the same stream (as per MPEG2 standard) are inherently synchronized.

All of the aforementioned constraints are sometimes colleitively referred to as the quality of presentation (QoP) [2]. The QoP should not be confused with network QoS, which includes network-related parameters such as maximum packet rate. packet loss probability, packet delay, and packet delay variation.

The frame streams within a presentation can be synchronized at the server before sending it to the user. However, frames are subject to varying delay in the network, hence the streams must be re-synchronized upon reception.

At the receiving end, the client system must synchronize the data both within a single stream and between different streams (e.g., audio, video, and subtitle text streams of a movie), and present them to the user in a configurable spatial arrangement.

In most cases, the client does not wait for all presentation data to arrive before starting to display them [6] and some- times the presentation must run in real time (e.g., multimedia conferencing). Hence, buffering capabilities of the client are not of primary importance, even though some buffering is usually required in order to compensate for possible objectlevel jitter, especially if the components of the presentation come from different sources.

\section{Errors and tests}

Errors in a DMSS may be categcrized $i:$ fun tional errors, timing (or component-level synchronization) errors, and synchronization errors (at the frame level), denoted by $F, T$, and $S$, respectively.

To illustrate the error categorization in more detail, we will list some of the more common errors found in DMSS.

- The connection may not be established properly, or it may not be established at all (class $F$ ).

- The server may fail to generate proper components of a presentation (class $F$ ), or the proper component timing (class $T$ ).

- The server(s) may generate proper streams, but with distorted frame timing and/or synchronization (class $S$ ). It may also fail to generate all frames required for a particular stream (class $S$ ). 


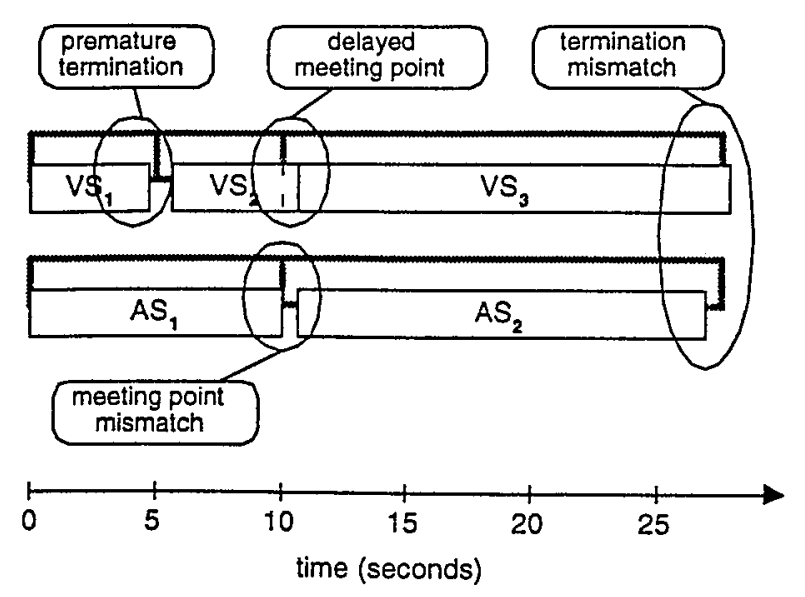

(a)

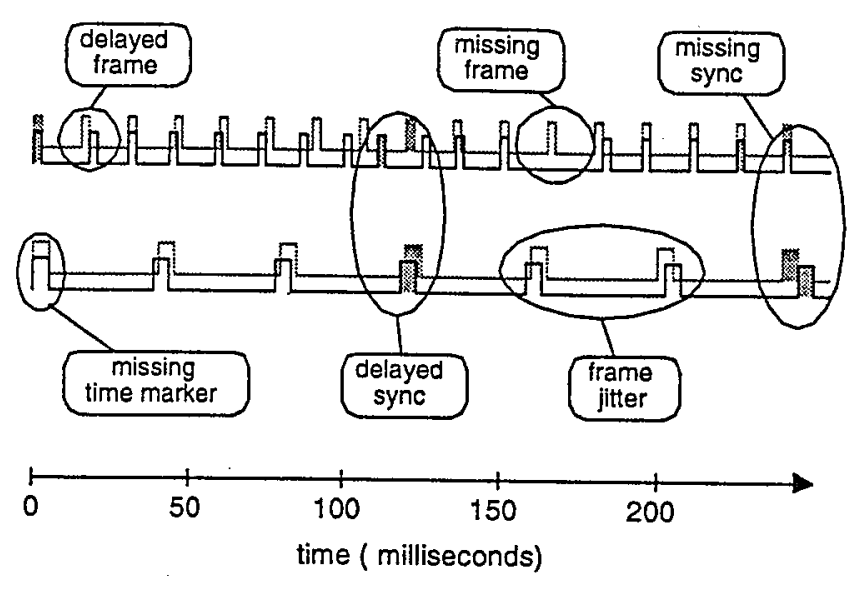

(b)

Figure 2. Errors: (a) component-level and (b) frame-level. The ideal schedules are shown in gray.

- The interconnection network may introduce several types of errors:

- Frames arrive to their destination with a variable delay (class $S$ ), which must be compensated for by the client.

- Presentations from multiple servers have different (and variable) delays which may affect synchronization, at both the coarse- (component) and fine-grain (frame) levels (classes $T$ and $S$ ).

- Frames can also be lost under certain conditions, depending on the network load and the mechanism of collision handling used by the network, which can give rise to $S$ class errors.

Other errors related to data transmission, such as corrupted fiames, are assumed to be handled by the underlying network layers, and thus are outside the scope of our testing framework.

- The packet jitter may be introduced at the client as well ( $S$ class). The scheduling policy of the client's operating system may introduce processing delays and thus impair the performance (and sometimes even the functionality) of the client. The problem will be even more pronounced if the client operating system is not capable of real-time operation or does not make use of preemptive scheduling [16], which will lead to nonpredictable delays (a majority of home computers today do not run real-time operating systems).

- The client may fail to satisfy either its functional requirements (class $F$ ), or its timing/synchronization requirements (classes $T$ and $S$ ). Examples of such errors include playing the wrong streams, erroneous spatial arrangement, and missing or incorrect synchronization.

- Reaction to user commands may be missing, delayed, or erroneous (class $F$ ).

Some examples of the last two classes of errors are shown in Fig. 2. Note that both $S$ and $T$ class errors may be related to a single stream, or to the interaction between multiple streams.

We consider the errors in the connection and termination phase to be predominantly functional, as no actual data transfer takes place. On the other hand, in the presentation phase (as well as in the dynamic reconfiguration phase), errors of all three types may appear.

The QoP.management policy in the presentation phase depends on the system parameters as well as on the agreement between the service provider (server and network part of the system) and the client. The issues related to QoP management are important research topics in the area of multimedia systems [9], and work has been reported on QoP modeling and specification [5].

Test classification may follow the above error classification, hence the tests may be categorized into three groups.

- Functional tests cover user control actions such as presentation start, stop, restart, pause, reverse the direction of the playout, fast forward and fast rewind, and skip to a specified time instant.

- Timing tests should concentrate on component-level 


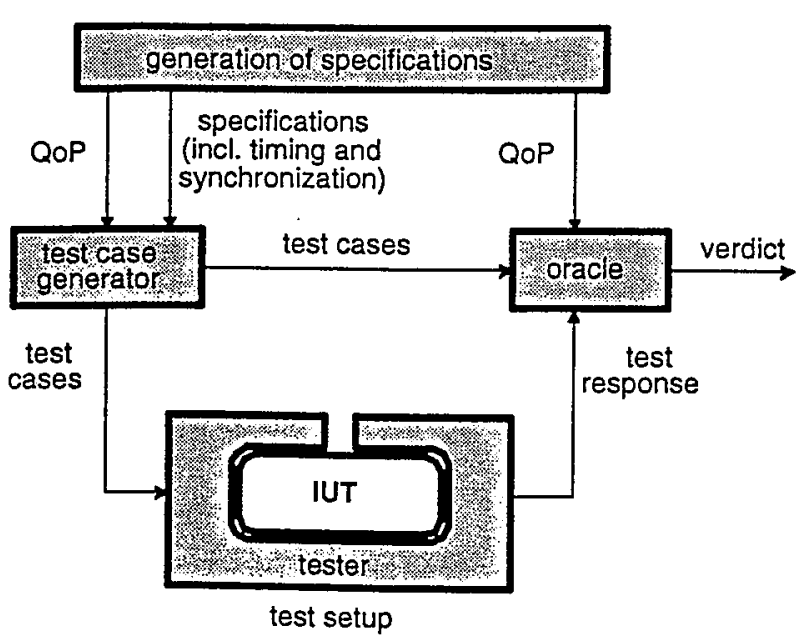

Figure 3. General test architecture for multimedia software systems.

timing, with presentations having one, two, or more components (both stream- and non-stream-oriented).

- Synchronization tests cover the intra- and interstream synchronization capabilities, using presentations with one or more stream-based components.

As different types of tests cover different aspects of the presentation, several tests may be conducted simultaneously using a single presentation.

\section{Testing of distributed multimedia software}

The test system consists of the following components:

- Specification generator, which provides specifications of presentations to be used for test case generation.

- Test case generator, which generates the test cases and provides information on the coverage and fault detection capabilities of the test.

- Test setup, which includes the tester and the implementation-under-test (IUT). The tester executes the test cases and monitors the IUT responses.

- Oracle, which compares the outputs of the IUT with the expected responses, and provides the verdict, i.e., decides whether the IUT has passed or failed the test.

The components and their relationships are shown schematically in Fig. 3.
As mentioned, DMSS testing is focused on timing and synchronization properties. We shall assume the IUT has already been thoroughly checked for functional correctness using standard functional testing techniques [3].

The configuration of the test setup for a distributed system is presented in Fig. 4 (a). For simplicity, only one server and one client are shown. Components of the tester are coloured in light gray. Thin arrows denote commands, while wide arrows denote stream data. In order to distinguish between 'real' (i.e., IUT-generated) and 'simulated' (i.e., tester-generated) data, the former is represented by black arrows, while the latter is denoted with dark gray ones.

For the server, simulated client commands are used as test inputs, while the data streams generated in response to these commands are monitored as outputs. On the other hand, the client input consists of one or more data streams with predefined characteristics, and the client output consists of the actual displayable data streams. These streams are monitored, and their pertinent properties recorded by the tester.

In both cases, the measurement of elapsed (absolute) time with adequate resolution and accuracy is required, and may involve timestamping and clock synchronization. The details are beyond the scope of this paper.

Although this configuration follows the established principles of conformance testing [11], it is inadequate for testing of distributed multimedia systems. First, distributed systems are tested with the aid of distributed testers, which may be difficult to control and synchronize. Second, the system under test includes the network. A real shared public network (such as the Internet) is difficult to control and the delay times are nondeterministic. The results obtained can be very much dependent on the network load at the time of testing. A private network could be used in order to obtain more stable results. However, it is more expensive to use and not really necessary. The network could be simulated and replaced with the appropriate tester extension as shown in Fig. 4 (b). This may seem to compromise the quality and coverage of the test since we are not testing the system in its operational environment. However, this architecture allows far better control of the network parameters, as packet delay and jitter (and the associated frame delay and jitter) can be varied at will. Moreover, other forms of erroneous network behavior-which are hard to control in a real network - can be simulated with ease. Finally, the use of the tester to simulate the network eliminates the need for distributed testers, and centralized testers are much easier to implement and operate.

It may be argued that such an architecture requiresa more complex tester system. Namely, the input streams (which are normally generated by appropriate server(s) and transmitted through the network) must be generated by the tester instead, including the distortion normally introduced in a 


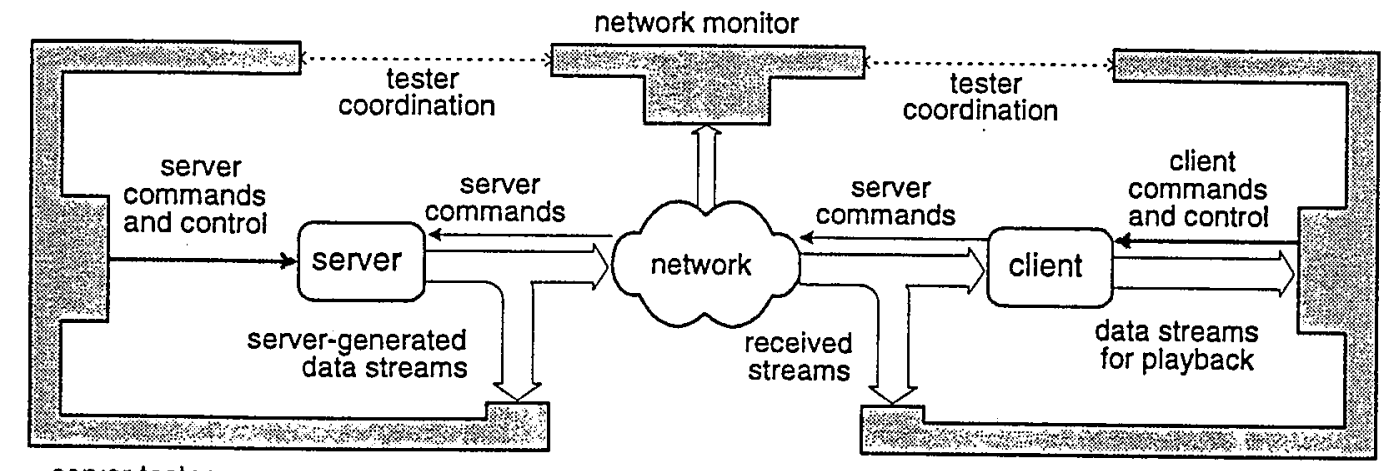

server tester

(a)

client tester

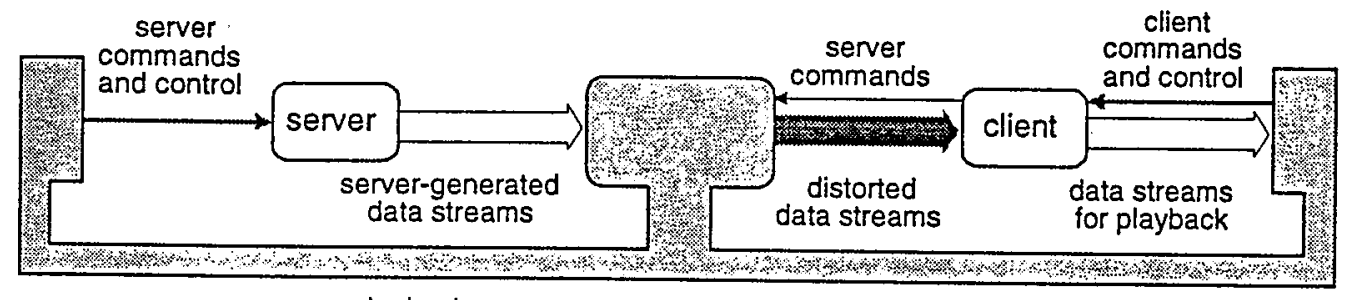

(b)

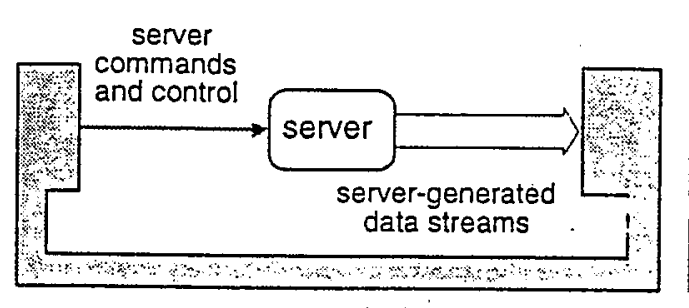

server test setup

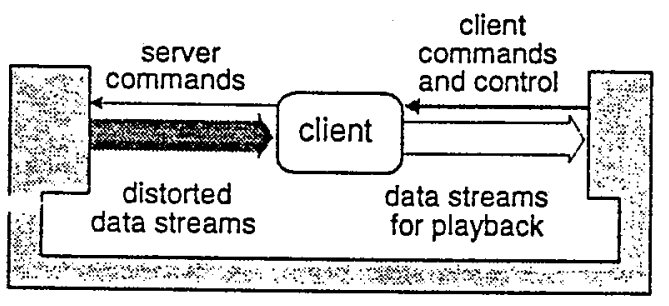

client test setup

(c)

Figure 4. Different test setup configurations. 
real network. The tester could synthesize these streams, or just play out streams saved during a previous session. In both cases, however, the increase in tester complexity is not high, and, given the increased controllability and the possibility of centralized testing, it certainly is worth the effort.

This architecture can be further simplified by separating the testing of the server and client as shown in Fig. 4 (c). In this architecture, all activities are much simpler, including the design of the testers, the design and generation of test cases, the control of test runs, and the analysis of test results. Therefore, it is this architecture that will be used as the basis for further considerations.

\section{Test case generation}

We have seen that a single presentation can often be used as the test case for both $T$ and $S$ errors - the difference being mainly in the analysis and interpretation of test results. Moreover, most presentations can serve as the basis for $F$ class tests as well. In the following, we will assume that a number of stream- and nonstream-based components are available, and discuss the criteria for choosing the test cases in more detail. Test cases are specified with a set of presentation components and a set of temporal relationship constraints between pairs of components.

$$
\begin{aligned}
& T C_{i}::=\left\{\left\{C_{1}, C_{2}, \ldots\right\},\right. \\
&\left\{\left(C_{i}, C_{j}\right), O p, t\right\}, \\
& \vdots \\
&\}
\end{aligned}
$$

(The presentation components are denoted by $C$, the temporal relationship operators by $O p$, and the time delay values by $t$.)

The most important criterion for test case selection is completeness: the set of test cases should be cover all static and dynamic properties the IUT is expected to satisfy. Therefore, tests could start with presentations with a single component, and proceed to presentations containing multiple components, exercising all meaningful combinations of appropriate temporal operators. For each operator, a set of meaningful values of the time delay should be chosen. Obviously, the number of test cases can be very large even for moderately small number of components and time delay values; note that the number of possible operators is fixed - see Table 1.

On the other hand, testing is always constrained by time and cost - i.e., it should consume the least possible amount of time and resources. Unfortunately, this contradicts with the above completeness criterion, which must be somewhat relaxed in order to keep the testing time and cost within reasonable bounds. Some of the relaxations are described in the following.

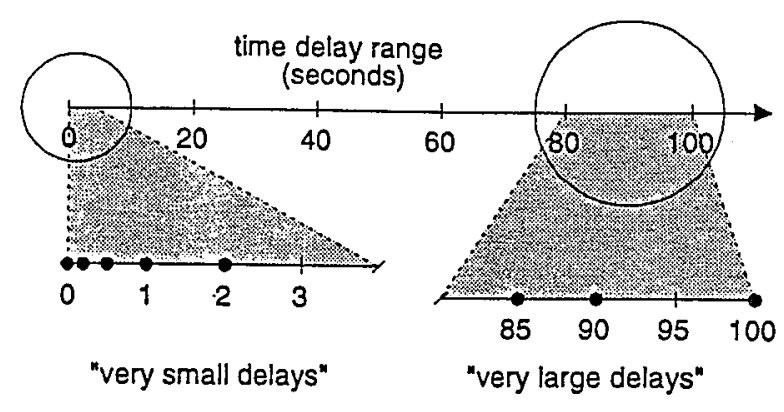

Figure 5. Small and large time delay values: chosen values shown as dots.

The time delay values mentioned above refer to the temporal relationships at the component level. The range of values allowed and the resolution used mainly depend on two factors:

- the durations of individual components (e.g., a delay of five minutes between two five-second clips is somewhat too long to be significant), and

- human perception abilities, which pose limits on both ends of the range. For ecample, a time delay between two components ( $A$ before $B$ by $t$ ) of less than, say fifty milliseconds is hardly noticeable. On the other extreme, an audio clip shown an hour after a video clip could hardly be considered as a valid two-component presentation).

The number of actual values used should be reduced as much as possible, with some of the time values very small, and others very large. In this context, 'small' and 'large' refer to the extreme values within the possible time delay range used for multimedia presentations. Since we are considering the component-level relationships, the range could be from zero to several minutes. Smaller delay values (i.e., those close to zero) are expected to impose more work on the IUT and, therefore, more likely to expose errors than the larger ones. Consequently, smaller values should receive more attention, i.e., more delay values should be chosen close to the bottom of the delay range. Normally, a few values close to 0, say at several hundred millisecond intervals, plus a few values at the high end of the chosen range, should be sufficient. An example time delay interval-with some randomly chosen delay values shown as dots-is given in Fig. 5.

Second, note that the temporal operators with relative timing cannot really be tested, since test cases must contain some specific values. Instead, they will be subsumed by equivalent operators which are specified with absolute 


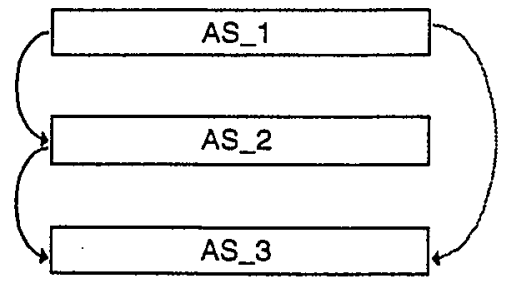

AS_1 equals AS_2: PASS and

AS_2 equals AS_3: PASS

Figure 6. Operator transitivity helps reduce the number of test cases.

timing. For example, if the presentation component $\mathrm{A}$ is required to end at an unspecified time before the start of component $\mathrm{B}$, it makes little difference whether the unspecified time assumes a value of $0.5 \mathrm{~s}$ or $1.5 \mathrm{~s}$. Hence, a system that passes the test with an absolute timing operator "A before $\mathrm{B}$ by $t$ ' for any value of $t$, may safely be assumed to pass the test with the relative timing operator ' $A$ before $B$ ' as well.

Following a similar argument, the operators with implicit absolute timing may be regarded as special cases of those with explicit absolute timing. For example, the operator 'A meets $B$ ' is equivalent to 'A before $B$ by 0 '. (The only exception is the 'equals' operator: 'A equals $B$ ' means that 'A starts with B' and, simultaneously, 'A ends with B'.) This will reduce the number of distinct operators to be tested even further.

The number of test cases can be reduced even further if we assume that multimedia presentations exhibit the 'transitivity' property with respect to temporal operators, which will be illustrated with an example. Consider, for example, the relationship 'A equals B', and a presentation with three components, say AS_1, AS_2, and AS_3, shown schematically in Fig. 6. If the IUT passes the test cases which contain 'AS_l equals AS_2' and 'AS.2 equals AS_3', it would be safe to conclude that it will pass the test case containing 'AS_l equals AS_3' as well. Therefore, instead of testing all possible pairs of components, we need to test all pairs such that every component appears at least once. In this manner, we could reduce the number of component pairs to be tested with 'equals' operator from $\frac{n(n-1)}{2}$ to just $n-1$ (where $n$ is the number of components in the test presentation). Similar arguments can be found for other temporal operators, giving rise to significant savings in the number of distinct operators to be used, and, consequently, in the number of test cases.

As for the choice of presentation components, it is reason- able to assume that non-stream-oriented components will be easier to generate, transmit, and play back than the streamoriented ones.

Furthermore, if the presentation includes several components of the same types, there is no need to test all possible pairs. It will suffice to limit the testing to different pairs such that all component types present are exercised. In more formal terms, given the two test cases

$$
\begin{aligned}
T C_{1}= & \left\{\left\{C_{1}, C_{2}\right\}\right. \\
& \left.\left\{\left(C_{1}, C_{2}\right), O p, t\right\}\right\} \\
T C_{2}=\{ & \left\{\left\{C_{3}, C_{4}\right\},\right. \\
& \left.\left\{\left(C_{3}, C_{4}\right), O p, t\right\}\right\}
\end{aligned}
$$

if $C_{3}$ and $C_{1}$ are of the same type (e.g., both are audio streams), and the same holds for $C_{2}$ and $C_{4}$ (which may be, say, video streams), then the latter test case, $T C_{2}$, is subsumed by the former, $T C_{1}$, and can be dropped. For example, assume that a total of five components are available, of which there are three audio and two video clips, and we want to make a two-component presentation. Then, instead of 10 possible combinations, we need to test only three, and still obtain the coverage of all possible pairs of component types. Of course, the reduction will be greater with more components: 3 instead of 10 for three-component presentations, and 2 instead of 5 for four-component presentation (the reduction does not apply to five-component presentations, of which there is only one in either case). The total number of component combinations is thus reduced to below $40 \%$ of the original ( 9 vs. 26).

All of the above arguments are valid for tests of the $T$ class. Tests of the $S$ class follow a different pattern of complexity growth. As noted before, one stream is usually used as the reference to which all others are synchronized. Hence, the number of possible component combinations is equal to the number of components used, which translates into a linear growth with the number of components. (Note, however, that the increase in number of streams actually shifts the foclis of the test towards performance, rather than synchronization, issues.) Actually, these tests can be combined with the tests of the $T$ class using the same presentations as test cases for both (see next section).

Finally, we note that most tests require measurement of the server response time, for which almost any presentation would do; but presentations with multiple components are more likely to expose errors of this type. The client response time is less important in offline applications, but for some real-time applications such as multimedia conferencing, response time is a crucial parameter.

Server performance could be tested as well by following the procedure outlined below, except that multiple presentations should be run simultaneously. The number of presentations can be gradually increased during the test; the dependence of the frame jitter on the number of presenta- 
tions could serve as a measure of server performance (other measures are also possible). Presentation schedules from previous test runs could be reused in subsequent runs. The client part of the system is less critical with respect to performance, since a client is seldom required to display two or more presentations at any given time. However, if a system performs a dual role, being both a client and a server at the same time, then performance issues may become crucial, and additional test cases may be required.

Note that it is possible to create a generic test suit which tests all meaningful pairs of multimedia objects related by a temporal operator as discussed above. All DMSS clients and servers should pass the generic tests as well as scenarios specifically created for a particular IUT based on the higherlevel functions supported by that IUT.

\section{Test procedures}

\subsection{Server testing} steps.

The procedure of server testing consists of the following

\section{PROCEDURE 1: SERVER TESTING.}

1 for each test case

1 create/select a presentation schedule

2 check consistency of the chosen schedule

3 select user action(s)

4 check applicability and feasibility of user action(s)

5 select values for different QoP parameters

$6 \quad$ calculate expected frame timing

7 transform the schedule into

a sequence of server commands

8 exercise the IUT and collect the test results

9 analyze results and give verdict

Note that steps 2,3, and 4 are optional.

The following cumments are in order.

The test designer can create a schedule for the test; alternatively, a number of predefined schedules may be made available, and the designer would have to select one of them for each test case.

The selected schedule may not be realizable: not all combinations of temporal relationship operators are meaningful, and the synchronization may not be specified correctly. (That is why the consistency check in step 2 may be necessary.) However, an automated tool which generates presentation schedules suitable for testing could easily perform all the necessary checks.

The user actions may be simulated at predefined time instants; alternatively, the system may be configured to generate these actions randomly. However, not all commands make sense for every component type, and each command
Table 2. An example of nominal and observed frame schedules for server testing.

\begin{tabular}{cccc} 
& & \multicolumn{2}{c}{ time [ms] } \\
stream id. & frame no. & expected & observed \\
\hline AS_1 & 01 & 0 & 120 \\
VS_1 & 01 & 0 & 232 \\
$\ldots$. & $\ldots$ & $\ldots$ & $\ldots$ \\
VS_1 & 12 & 440 & 642 \\
AS_1 & 31 & 450 & 650 \\
AS_1 & 32 & 465 & 664 \\
AS_1 & 33 & 480 & 682 \\
VS_1 & 13 & 480 & 684 \\
$\ldots .$. & $\ldots$ & $\ldots$ & $\ldots$
\end{tabular}

must be allowed sufficient time to be executed; hence the selected actions must be checked for applicability and/or realizability (step 4).

'Expected frame timing' in step 6 consists of the information about the type of the generated frame and the time (relative to the beginning of the presentation) when it should appear at the output. A sample excerpt from the schedule is shown in Table 2, with frame types, frame numbers, and expected times shown.in the first three columns, respectively. The times are calculated relative to the start of presentation, assuming $40 \mathrm{~ms}$ and $15 \mathrm{~ms}$ periods (i.e., 25 and $66.67 \mathrm{~Hz}$ frame rates) for video and audio frames, respectively.

The sequence of server commands created in step 7 is used as server input. Note that all actions up to and including step 7 can be performed off-line, and the calculated schedules and the appropriate server commands can be stored for later use. The tester sends the commands to the server, and monitors the server output. (Observed output frame timing is shown in the last column of Table 2.) The oracle compares the observed schedules with the expected ones. If the differences do not exceed the limits imposed by the QoP parameters, the verdict is PAsS, otherwise it is FAIL. For example, if the jitter of the audio stream at the server output must be less than $2 \mathrm{~ms}$, the sample test case shown would FAlL because of the $18 \mathrm{~ms}$ delay between frames 32 and 33 .

By examining the first and the last frames of each component, the oracle can also give the verdict on componentlevel timing. For example, assume that the componentlevel schedule reads 'VS_l startwith AS_l', as shown in Fig. 1 (c). In the sample test case shown, the time difference between the first frames of AS_1 and VS_l is $112 \mathrm{~ms}$. Then, the verdict would be FAlL if the QoP-required tolerance for this operator is $100 \mathrm{~ms}$, and PASS if this tolerance is $150 \mathrm{~ms}$. - Note that test cases for both $T$ and $S$ class tests need not contain meaningful presentation data, as the analysis of test 
results does not require human involvement. However, the actual content of the data streams still has to be examined for correctness, especially if the test case includes user interactions, where the same data may be output at different rates and/or with different ordering.

\subsection{Client testing}

Two main differences may be observed that distinguish client and server testing: the nature of test inputs and the number of test cases. Test inputs for server testing are high-level commands, while for client testing they are frame streams. As for the number of test cases, a test case for server testing corresponds to a single set of server commands, and it is executed only once. For client testing, each presentation schedule is used to generate several (slightly different) input frame streams, with each stream being a separate test case.

The client testing procedure may again be partitioned into a number of steps, as described by the following procedure.

\section{PROCEDURE 2: CLIENT TESTING. \\ for each set of test cases \\ 1. create/select a presentation schedule \\ 2 check consistency of the chosen schedule \\ 3 select user action(s) \\ 4 check applicability and feasibility of user action(s) \\ 5 select values for different QoP parameters \\ $6 \quad$ calculate expected frame timing \\ 7 for each test case \\ $7.1 \quad$ calculate input frame timing \\ 7.2 transform the schedule into \\ sequence of server commands \\ 7.3 exercise the IUT and collect the test results \\ $7.4 \quad$ analyze results and give verdict}

(As before, steps 2, 3, and 4 are optional.)

If the set of test cases includes user actions (and appropriate commands), a reasonable value (or range of values) for server response time must be chosen as well. This time must be taken into account in calculating the expected frame timing.

The expected frame timing is calculated in the same manner as for server testing. In step 7.1 - first in the inner loop, the expected frame times are modified by adding a variable delay, which models the jitter introduced by the network. The distribution of the delay values may be chosen to model different patterns of network behavior. For each pass through the loop, a different frame schedule will be produced - an example is shown in Table 3. (As before, the times are calculated relative to the start of presentation, assuming $40 \mathrm{~ms}$ and $15 \mathrm{~ms}$ periods for video and audjo frames, respectively.) At the client output, the re-generated streams are monitored and their timing is compared with the
Table 3. An example of a calculated frame schedule for client testing.

\begin{tabular}{cccc} 
& & \multicolumn{2}{c}{ time [ms] } \\
stream id. & frame no. & expected & with jitter \\
\hline$\ldots$ & $\ldots$ & $\ldots$ & $\ldots$ \\
VS_1 & 12 & 440 & 445 \\
AS_1 & 31 & 450 & 453 \\
AS_1 & 32 & 465 & 466 \\
AS_1 & 33 & 480 & 479 \\
VS_1 & 13 & 480 & 482 \\
$\ldots$ & $\ldots$ & $\ldots$ & $\ldots$
\end{tabular}

nominal schedule. Again, all calculations can be performed off-line, prior to actual testing, and the calculated schedules may be stored for later use.

In order to test for client robustness, the delay times may be made larger than the maximum values prescribed for client tolerance. In this manner, we can check how much distortion of the input data timing is the client system able to tolerate, while still producing correct output.

\section{Conclusions and directions for further re- search}

The proposed methodology presents a conceptual framework showing how DMSS can be tested. In particular, tools need to be designed to facilitate test case generation at the schedule and frame level, and translation of test presentation schedules to appropriate client and server commands. The actual testing can be conducted either using specialized hardware testers, or by modifying appropriate software support libraries. Implementing the tests in software would indeed incur higher overhead, but at a significantly lower cost compared to the hardware-based solution.

We note that the testing of distributed multimedia systems could benefit from improvements in techniques for specifying timing and synchronization constraints as well as user interactions.

We are currently implementing a software prototype of the proposed framework, and the results and experiences learned will be reported in a subsequent paper.

\section{References}

[1] J. F. Allen. Maintaining knowledge about temporal intervals. Communications ACM, 26(11):832-843, 1986.

[2] S. Baqai, M. F. Khan, M. Woo, S. Shinkai, A. A. Khokhar, and A. Ghafoor. Quality-based evaluation of multimedia 
synchronization protocols for distributed multimedia information systems. IEEE Journal of Selected Areas in Communications, 14(7):1388-1403, Sept. 1996.

[3] B. Beizer. Software Testing Techniques. Van Nostrand Reinhold, New York, 2nd edition, 1990.

[4] A. C. Coombes and J. A. McDermid. Specifying temporal requirements for distributed real-time systems in Z. Software Engineering Journal, 8(5):273-283, Sept. 1993.

[5] J. de Meer and B. Sarikaya. Quality of service modelling of continuous media system behaviour. Journal of Electrical and Electronics Engineering, Australia, 16(1):45-56, Mar. 1996.

[6] D. Ferrari. Client requirements for real-time communication services. IEEE Communications Magazine, 28(11):65-72, Nov. 1990.

[7] D. Fibush. Testing multimedia transmission systems. IEEE Design \& Test of Computers, 12(4):34-44, Winter 1995

[8] C. Gaibisso, G. Gambosi, M. Lancia, and M. Vitale. Multimedia conferencing on packet switched networks: testing and evaluation. Computer Networks and ISDN Systems, 26 (suppl.)(2-3):S139-S146, 1994.

[9] J. Grabowski and T. Walter. Testing Quality-of-Service aspects in multimedia applications. In Proceedings of the 2 nd Worskhop on Protocols for Multimedia Systems (PROMS), Oct. 1995.
[10] ISO/IEC. Information Technology - Generic coding of moving pictures and associated audio information - Part 1: System; Par 2: Video. International Standard 13818, 1994.

[11] ISO/IEC JTC 1/SC $21 \mathrm{~N}$. Information Technology - Open System Interconnection - Conformance Testing Methodology and Framework - Part 1-5. International Standard 9646. 1991.

[12] L. Jin, H. Zhu, and P. Hall. Adequate testing of hypertext applications. Information and Software Technology, 39(4):225-234, Apr. 1997.

[13] T. D. C. Little and A. Ghafoor. Synchronization and storage models for multimedia objects. IEEE Journal of Selected Areas in Communications, 8(3):413-427, Apr. 1990.

[14] M. Ramachandran. Testing interactive multimedia systems for quality. In I. Marshall, W. Samson, and D. Edgar-Nevill, editors, Proceedings of the 4th Software Quality Conference, volume 2, pages 289-299, July 1995.

[15] B. Sarikaya. Principles of Protocol Engineering and Conformance Testing. Ellis Horwood, New York, NY, 1993.

[16] J.-J. Sung, M.-Y. Huh, H.-J. Kim, and J.-H. Hahm. Hypermedia information retrieval system using MHEG coded representation in a networked environment. In R. Steinmetz, editor, Multimedia: Advanced Teleservices and High-Speed Communication Architectures, 2nd International Workshop IWACA '94, pages 67-77, Heidelberg, Germany, Sept. 1994. Springer-Verlag. 